\title{
Debrominated, hydroxylated and methoxylated metabolism in maize (Zea mays L.) exposed to lesser polybrominated diphenyl ethers (PBDEs)
}

\author{
Sen Wang ${ }^{\mathrm{a}, \mathrm{b}}$, Shuzhen Zhang ${ }^{\mathrm{a}, *}$, Honglin Huang ${ }^{\mathrm{a}}$, Anxiang Lu ${ }^{\mathrm{c}}$, Hua Ping ${ }^{\mathrm{c}}$ \\ a State Key Laboratory of Environmental Chemistry and Ecotoxicology, Research Center for Eco-Environmental Sciences, Chinese Academy of Sciences, P.0. Box 2871, \\ Beijing 100085, China \\ ${ }^{\mathrm{b}}$ Department of Environmental Sciences, College of Urban and Environmental Sciences, Northwest University, Xi'an 710027, China \\ ${ }^{\mathrm{c}}$ Beijing Research Center for Agri-Food Testing and Farmland Monitoring, P.O. Box 2449-26, Beijing 100097, China
}

\section{H I G H L I G H T S}

- Debromination, hydroxylation and methoxylation of PBDEs in plant occurred rapidly.

- The introduction of a hydroxyl/methoxy group at the ortho-positions was easier.

- PBDEs are more susceptible to metabolism in leaves and stems than in roots.

- $\sum \mathrm{MeO}-\mathrm{PBDEs}$ concentration was significantly higher than $\sum \mathrm{OH}-\mathrm{PBDEs}$ concentration.

\section{A R T I C L E I N F O}

\section{Article history:}

Received 7 February 2012

Received in revised form 14 May 2012

Accepted 15 May 2012

Available online 8 June 2012

\section{Keywords:}

PBDEs

Plant metabolism

Debromination

Hydroxylation

Methoxylation

\begin{abstract}
A B S T R A C T
A hydroponic experiment was conducted to investigate the debrominated, hydroxylated and methoxylated metabolism of polybrominated diphenyl ethers (PBDEs, BDE-15, -28 and -47) in maize. A total of six debrominated metabolites (de-PBDEs), seven hydroxylated PBDEs (OH-PBDEs, including two unidentified OH-di-PBDEs and one unidentified OH-tri-PBDE) and four methoxylated PBDEs (MeO-PBDEs) were determined in the exposed plants. The metabolic products were detected in maize only after $12 \mathrm{~h}$ of exposure to the PBDEs. However, the concentration of each type of the metabolites (de-PBDEs, OH-PBDEs or MeOPBDEs) decreased at the later exposure time, possibly due to further metabolism. The removal of a bromine atom or the introduction of a hydroxyl/methoxy group was easier at the ortho-positions on the biphenyl structure than at the para-positions. Concentration ratios of the total debrominated, hydroxylated or methoxylated metabolites to the parent congener (BDE-28 or -47) generally followed the order of leaves $>$ stems $\gg$ roots, and MeO-PBDEs $>$ de-PBDEs $\gg$ OH-PBDEs. These results suggest that metabolism occurred preferentially in leaves and stems than in roots. Less transformation and shorter elimination half-life of OH-PBDEs would contribute to the lower concentrations of OH-PBDEs than of de-PBDEs or MeO-PBDEs in maize.
\end{abstract}

(c) 2012 Elsevier Ltd. All rights reserved.

\section{Introduction}

Polybrominated diphenyl ethers (PBDEs) are widely used as new additive brominated flame retardants (de Wit, 2002). Owing to their high volume production, lipophilicity and persistence, PBDEs have become ubiquitous contaminants in the environment (UNEP, 2002; Hassanin et al., 2004; Hites, 2004). Since May 2009 commercial mixtures of penta- and octa-BDEs have been included on the Stockholm Convention list of priority persistent organic pollutants due to their high bioaccumulation and potential toxicity (California State Assembly, 2003; Directive EEC, 2003).

\footnotetext{
* Corresponding author. Tel.: +86 10 62849683; fax: +86 1062923563 .

E-mail address: szzhang@rcees.ac.cn (S. Zhang).
}

Degradation of PBDEs is an important process which determines their fate in the environment. PBDEs have been shown to break down into lower brominated congeners (de-PBDEs) with much higher toxicity by microbial (He et al., 2006; Betts, 2008; Robrock et al., 2008), photochemical (Eriksson et al., 2004; Shih and Wang, 2009), and plant degradation (Huang et al., 2010; Wang et al., 2011b). Hydroxylated PBDEs (OH-PBDEs) and methoxylated PBDEs (MeO-PBDEs) have been detected in marine organisms (algae, mussels, and fish), human blood, and abiotic samples (surface water, snow and rain) (Marsh et al., 2004; Sinkkonen et al., 2004; Malmvärn et al., 2005; Verreault et al., 2005; Ueno et al., 2008; Wang et al., 2011d). There are different opinions about the origin of OH-PBDEs and MeO-PBDEs in biota and abiotic environmental matrices. Some studies have demonstrated that 
OH-PBDEs are formed by hydroxylation of PBDEs or demethylation of MeO-PBDEs, and MeO-PBDEs are formed by methylation of the OH-PBDEs (Haglund et al., 1997; Teuten et al., 2005; Wan et al., 2009). Formation of OH-PBDEs and MeO-PBDEs in animals after exposure to PBDEs has also been observed in laboratory studies (Kierkegaard et al., 2001; Malmberg et al., 2005; Qiu et al., 2007; Munschy et al., 2010). On the other hand, OH-PBDEs and $\mathrm{MeO}-\mathrm{PBDEs}$ have been identified as marine natural products (Wan et al., 2009). Plants are an important component of the terrestrial ecosystem and play an important role in the metabolism of organic contaminants in the environment (Collins et al., 2006). However, there have been few studies on the metabolic occurrence and fate of PBDEs in plants (Huang et al., 2010; Wang et al., 2011a).

There has been a considerable interest in investigating the behavior of $\mathrm{OH}-\mathrm{PBDEs}$ in the environment owing to their greater toxicity than the parent PBDE congeners and the fact that they elicit a variety of effects on exposed organisms including disruption of thyroid hormone homeostasis, oxidative phosphorylation disruption, antiprogestagenic potency, altered estradiol synthesis, and neurotoxic effects (Meerts et al., 2001; Qiu et al., 2007; Wan et al., 2009). Although the toxicity of MeO-PBDEs is low, the demethylation products of the corresponding OH-PBDEs may have high toxicity (Wan et al., 2009). Therefore, MeO-PBDEs and $\mathrm{OH}-$ PBDEs represent potential risks to human and animal health (Lacorte and Ilonomou, 2009; Wan et al., 2009; Ren et al., 2010). Plant uptake and metabolism of organic chemicals are important and represent the first step of the food chain and it is very important to evaluate the potential exposure risks for the environment and human health. PBDEs have been detected in plants (Mueller et al., 2006; Huang et al., 2010; Wang et al., 2011b) and it is therefore essential to further study their metabolism in plants.

In the present work a hydroponic experiment was conducted to investigate the metabolism of lesser PBDEs (BDE-15, -28 and -47) in maize. De-PBDEs, OH-PBDEs and MeO-PBDEs in different parts of maize plants were analyzed after different exposure times in order to elucidate the metabolism of PBDEs in plants.

\section{Materials and methods}

\subsection{Chemicals and regents}

The following standards were purchased from AccuStandard (AccuStandard, New Haven, CT, USA): BDE-15, -28 and -47 , a standard solution of PBDEs containing 39 native congeners (mono- through hepta-PBDEs), OH-PBDE standards (2'-OH-BDE3, 3'-OH-BDE7, 4'-OH-BDE17, 3'-OH-BDE28, 3-OH-BDE47, 5-OH-BDE47 and 6-OH-BDE47) and MeO-PBDE standards (2'-MeO-BDE3, 3'-MeO-BDE7, 4'-MeO-BDE17, 3'-MeO-BDE28, 3-MeO-BDE47, 5-MeO-BDE47 and 6-MeO-BDE47). Their molecular structures are shown in Table S1 in the Supplementary material. Standards of ${ }^{13} \mathrm{C}-\mathrm{PCB}-141$ and ${ }^{13} \mathrm{C}-\mathrm{PCB}-208$ were purchased from Cambridge Isotope Laboratory (Andover, MA, USA). Anhydrous sodium sulfate $\left(\mathrm{Na}_{2} \mathrm{SO}_{4}\right)$, silica gel and alumina (100-200 mesh) were washed with hexane and used after heating overnight at $150^{\circ} \mathrm{C}$. Florisil (60-100 mesh, Acros Organics, Geel, Belgium) was activated at $450{ }^{\circ} \mathrm{C}$ for $12 \mathrm{~h}$ and deactivated by adding $1 \%(\mathrm{w} / \mathrm{w})$ water. All solvents used, i.e., methyl tert-butyl ether (MTBE), dichloromethane (DCM), hexane, methanol, acetonitrile, acetone, were of HPLC grade and purchased from Fisher Scientific (Fair Lawn, NJ, USA). Ultrapure water (18.2 M $\Omega$ ) used was obtained by using a Milli-Q system (Milli-Q Advantage A10, Millipore Corporation, Billerica, MA, USA). All other chemicals and reagents used were of reagent grade.

\subsection{Exposure experiment}

Maize (Zea mays L.) seeds were purchased from the Chinese Academy of Agricultural Sciences, Beijing, China. They were surface sterilized by soaking in a $3 \%(\mathrm{v} / \mathrm{v}) \mathrm{H}_{2} \mathrm{O}_{2}$ solution for $30 \mathrm{~min}$, rinsed with autoclaved deionized water and pre-germinated on moist filter paper in the dark for $4 \mathrm{~d}$. Seedlings were then selected and transplanted in containers of half Hoagland nutrient solution and cultivated under a controlled environment growth chamber at a light intensity of $250 \mu \mathrm{mol} \mathrm{m} \mathrm{m}^{-2} \mathrm{~s}^{-1}$ provided by supplementary illumination with a photoperiod of $14 \mathrm{~h} \mathrm{~d}^{-1}$, a $25 / 20^{\circ} \mathrm{C}$ day/night temperature regime, and a relative humidity of $60-70 \%$. Autoclaved deionized water was used to prepare Hoagland nutrient solution which was saturated with oxygen to minimize the metabolism of anaerobic microorganisms.

After growth for $9 \mathrm{~d}$, every three selected plant seedlings were transferred to an exposure container, a $150-\mathrm{mL}$ glass-stoppered flask enclosed in aluminum foil to eliminate photolysis of PBDEs. Exposure experiments with BDE-15, -28 and -47 were conducted separately with their concentrations at 19.5, 21.6 and $15.5 \mu \mathrm{g} \mathrm{L}^{-1}$, respectively, lower than their water solubility. Fresh exposure solutions were prepared with the standard solutions of BDE-15, 28 and -47 , dissolved in acetone (lower than $1 \%$ v/v, in the exposure solution). All these were conducted in a laminar flow hood. During plant exposure, approximately $10 \mathrm{~mL} \mathrm{~d}^{-1}$ of autoclaved deionized water saturated with oxygen was injected into the containers to compensate for the transpiration losses. Blank (without PBDE exposure) and unplanted containers were included as controls and all the treatments were set up in triplicate. The containers were positioned randomly and re-randomized every day.

\subsection{Sample preparation}

Maize plants were harvested at intervals of $12,24,48,96,144$, and 216 h. Root samples were first carefully rinsed with deionized water to remove any adhering PBDEs, and rinses were collected and combined into the exposure solutions. Then maize leaves, stems and roots were washed thoroughly with deionized water, blotted with filter paper and weighed. All the samples were then frozen at $-50{ }^{\circ} \mathrm{C}$ overnight, freeze-dried for $48 \mathrm{~h}$ in a lyophilizer (FD-1, Beijing Boyikang Instrument Ltd, Beijing, China), and weighed to determine their dry weights. The dried samples were finely chopped and stored in glass containers at $-20^{\circ} \mathrm{C}$ before chemical analysis.

\subsection{Extraction and analysis}

Extraction and cleanup of PBDEs and MeO-PBDEs were based on the methods of López et al. (2009) and Wang et al. (2011b), and the detail method is provided in the Supplementary material. ${ }^{13} \mathrm{C}-\mathrm{PCB}-$ 141 was added as surrogate standard to the samples prior to extraction and ${ }^{13} \mathrm{C}-\mathrm{PCB}-208$ was added to the final solutions as an internal standard. An Agilent 7890 GC-MS (5975 inert) (Agilent, Palo Alto, CA, USA ) and a HP-5MS column $(30 \mathrm{~m} \times 0.32 \mathrm{~mm}$ i.d., $0.25 \mu \mathrm{m}$ film thickness) (J \& W Scientific, Folsom, CA) were used for the analysis of PBDEs and MeO-PBDEs (Wang et al., 2011b). The GC oven temperature program for the determination of PBDEs was as follows: $1 \mathrm{~min}$ at $110^{\circ} \mathrm{C}$, first ramp at $30^{\circ} \mathrm{C} \mathrm{min}^{-1}$ to $200^{\circ} \mathrm{C}(1 \mathrm{~min}$ hold $)$, second ramp at $3^{\circ} \mathrm{C} \mathrm{min}^{-1}$ to $260^{\circ} \mathrm{C}$ ( $12.5 \mathrm{~min}$ hold), and third ramp at $20^{\circ} \mathrm{C} \mathrm{min}^{-1}$ to $295^{\circ} \mathrm{C}$ (20 min hold). For the determination of MeO-PBDEs, the oven temperature program had the following parameters: $110^{\circ} \mathrm{C}(1 \mathrm{~min}$ hold $)$, raised to $215^{\circ} \mathrm{C}$ at $15^{\circ} \mathrm{C} \mathrm{min}-1$ ( 1 min hold), finally at $270{ }^{\circ} \mathrm{C}$ at $2{ }^{\circ} \mathrm{C} \mathrm{min}^{-1}$ (16 min hold). The carrier gas was helium at a flow rate of $1.5 \mathrm{~mL} \mathrm{~min}^{-1}$. More detailed information is available in the Supplementary material. 
The extraction and cleanup procedures for OH-PBDEs in plants have been reported in our previous study (Wang et al., 2011a), and the detailed method is provided in the Supplementary material. A UPLC-MS/MS system was used to detect and quantify OHPBDEs. Chromatographic analyses were performed using ACQUITY UPLC system (Waters, Milford, MA, USA). A reversed-phase chromatography was performed by Waters ACQUITY UPLC BEH $\mathrm{C}_{18}$ column $(2.1 \mathrm{~mm} \times 100 \mathrm{~mm}$ i.d., $1.7 \mu \mathrm{m}$ particle size, Waters, Milford, MA, USA). A binary mixture with acetonitrile (A) and water (B) was modified and used as the mobile phase. A linear gradient with a flow rate of $0.4 \mathrm{~mL} \mathrm{~min}{ }^{-1}$ was applied in the following manner: 70\% B (0-0.8 min), 70-30\% B (0.8-5 min), 30-5\% B (5-7 min), 5\% B (7-9 min), 5-70\% B (9-10 min), 70\% B (10-13 min). Mass spectrometry analysis was carried out using a Waters Xevo TQ MS triple quadrupole mass spectrometer equipped with an ESI source (Waters, Milford, MA, USA). The details of the UPLC-MS/MS method are provided in the Supplementary material.

\subsection{Quality assurance and quality control}

Quality assurance protocol included the addition of surrogate standards and random injection of solvent blanks and standards. The limits of detection (LOD) were in the range of 25-52, 32-58 and $30-210 \mathrm{pg} \mathrm{g}^{-1}$ in plant tissues for all the PBDE, MeO-PBDE and OH-PBDE congeners $(n=6)$, respectively. Recoveries of the surrogate standards of ${ }^{13} \mathrm{C}$-PCB-141 were $70.6-95.8 \%$. Recoveries of PBDEs, MeO-PBDEs and OH-PBDEs were within the ranges 73.7$108.4 \%, 67.8-102.3 \%$ and $68.2-94.6 \%$ in the matrix spiked samples, respectively.

\subsection{Data analysis}

The data were subjected to statistical analysis by using the SPSS version 12.0 software package. All of the data represent the mean and standard deviation of triplicates.

\section{Results and discussion}

\subsection{Debrominated metabolites of PBDEs in maize}

Debrominated metabolites of PBDEs were detected in maize root, stem and leaf samples. A total of two, six and five de-PBDEs were determined in maize exposed to BDE-15, -28 and -47 , respectively, after exposure for $12 \mathrm{~h}$ (Fig. 1), indicative of a very rapid debrominated metabolism. Among these debrominated metabolites, BDE-2 and -3 had relatively high concentrations, suggesting that PBDEs had a high debrominated metabolic ability in maize. This phenomenon was also found in numerous previous studies. For example, studies showed that BDE-209 was debrominated to the lower PBDEs such as di- and tetra-PBDEs in sediments, soils and plants (La Guardia et al., 2007; Tokarz et al., 2008; Huang et al., 2010). No de-PBDE was detected in the hydroponic solutions with the exception of the BDE-28 exposure experiment in which BDE-2, $-12,-13,-15,-32$ and -37 were detected in the solutions before plant exposure. However, altogether they accounted for less than $0.8 \%$ of the amount of BDE-28 added, and this was much lower than the concentrations in the exposed maize (Table S3, Supplementary material). These results suggest the occurrence of debromination of PBDEs in maize.

BDE-15, -28 and -47 investigated in this study were congeners with bromine at the ortho- or para-positions. Based on the de-PBDEs detected, BDE-3, BDE-3, -15 and BDE-3, -15, -28 were the debrominated metabolites of BDE-15, -28 and -47 , BDE-2, BDE-2, $-12,-13,-32,-37$ and BDE-2, $-12,-13$ were the products of bromine atom rearrangement of BDE-15, -28 and -47 , respec-
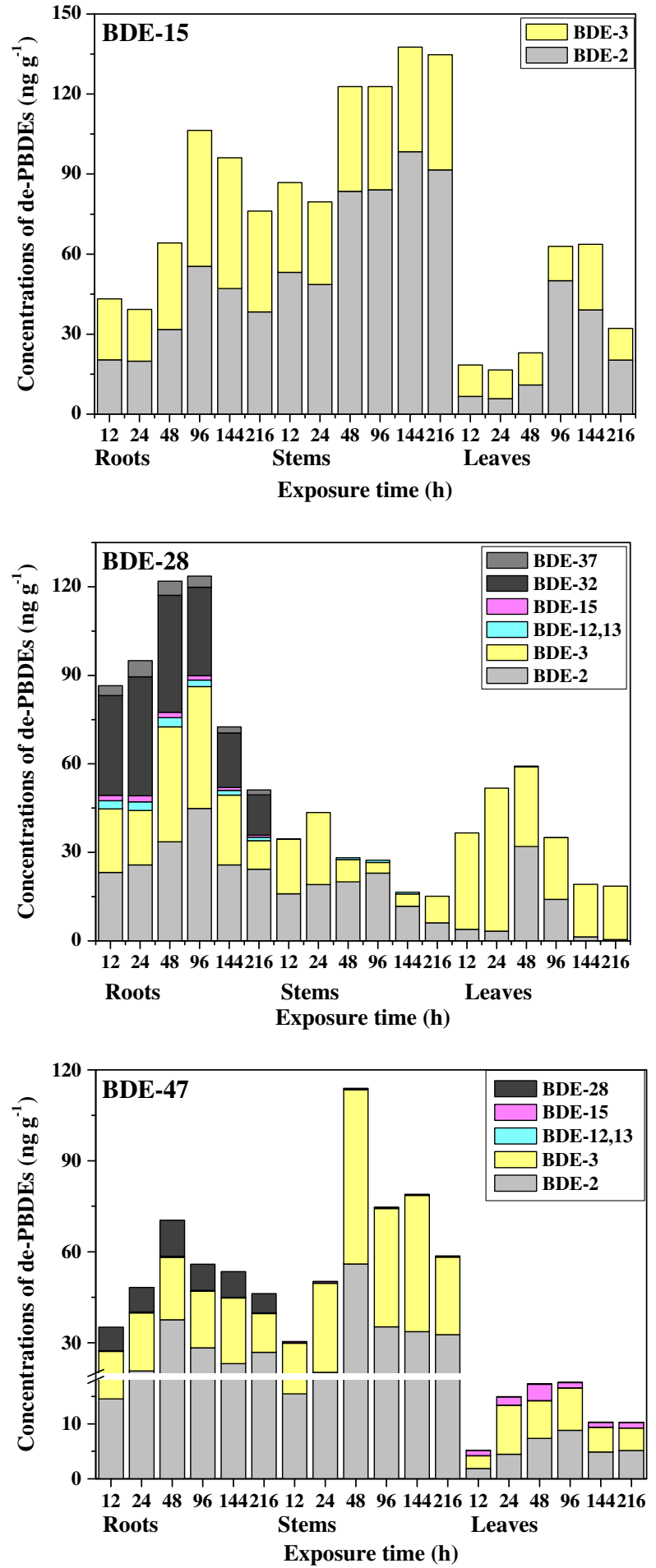

Fig. 1. Time-dependent concentrations of the debrominated PBDEs (de-PBDEs) in maize.

tively. In a previous study, chlorine dechlorination and rearrangement on the biphenyl rings were observed in poplars and switchgrass exposed to PCB-77 (Liu et al., 2009). However, no metabolite of the debromination at para-positions (BDE-8 and 17) was detected in the BDE-28 and -47 exposed maize, suggesting that bromine atoms at the ortho-positions departed more easily to form BDE-15 and -28. A previous study on the debromination metabolism of PBDEs in hexane under ultraviolet irradiation has shown that the bromine atoms at ortho-positions have much high- 
er elimination efficiency than those at meta- and para-positions (Fang et al., 2008). However, another study has demonstrated that the bromine atoms are preferentially removed from the meta- and para-positions on the biphenyl structure by microbial degradation (Gerecke et al., 2005).

Fig. 1 describes the time-dependent accumulation of de-PBDEs in maize. Accumulation of the de-PBDEs in each part of the maize plant generally increased firstly and then decreased to different extents for BDE-15, -28 and -47 . The decrease in accumulation may be caused by further metabolism to other lower brominated congeners or hydroxylated and methoxylated analogs. In comparison, the total concentration of the de-PBDEs was relatively low in leaves compared with roots and stems with the exception of a higher concentration in leaves than in stems of BDE-28 exposed maize. It was speculated that the lowest concentration of the parent PBDEs in leaves (Wang et al., 2011c) and the limited acropetal translocation of PBDEs inside plants with higher log $k_{\text {ow }}$ contributed to the lower concentrations of de-PBDEs in leaves than in roots or stems.

\subsection{Hydroxylated metabolites of PBDEs in maize}

Hydroxylated metabolites of BDE-15, -28 and -47 were analyzed in maize root, stem and leaf samples. A total of four OH-PBDEs of the seven OH-PBDE standards, two unidentified OH-di-PBDEs $(\mathrm{OH}-\mathrm{di}-\mathrm{BDE}(1)$ and $\mathrm{OH}-\mathrm{di}-\mathrm{BDE}(2))$ and one unidentified $\mathrm{OH}$-tri-PBDE were detected in maize (Fig. 2). All these metabolites were detected in maize only after exposure for $12 \mathrm{~h}$, indicating that hydroxylated metabolism in maize also occurred rapidly. Except that no OH-PBDE detected in the BDE-47 exposed maize leaf samples, two unidentified OH-di-PBDEs (OH-di-BDE(1) and $\mathrm{OH}$-di-BDE(2)), five OH-PBDEs (3'-OH-BDE28, 4'-OH-BDE17, $\mathrm{OH}-\mathrm{di}-\mathrm{BDE}(1), \mathrm{OH}-\mathrm{di}-\mathrm{BDE}(2)$ and one unidentified OH-tri-PBDE), and four OH-PBDEs (5-OH-BDE47, 6-OH-BDE47, OH-di-BDE(1) and $\mathrm{OH}-\mathrm{di}-\mathrm{BDE}(2))$ were detected in maize exposed to BDE-15, 28 and -47 , respectively. There were more types of $\mathrm{OH}-\mathrm{PBDE}$ congeners in roots than in stems or leaves. No OH-PBDE was measured in the blank maize controls (without PBDE exposure), suggesting that the OH-PBDEs detected were derived from metabolism of PBDEs in maize.

Among all the detected OH-PBDEs, the concentrations of the two unidentified OH-di-PBDEs(OH-di-BDE(1) and OH-di-BDE(2)) were higher than the other OH-PBDE congeners in the exposed maize, with the concentrations following the order: BDE$15 \gg$ BDE-28 > BDE-47. Furthermore, for di-PBDEs metabolites, the BDE-15 concentration was much higher than BDE-12 or -13 , and it is speculated that these two OH-di-PBDEs were OH-BDE15. In the BDE-47 exposed maize roots, the concentration of $6-\mathrm{OH}-$ BDE47 was higher than that of 5-OH-BDE47 and no 3-OH-BDE47 was detected, which suggests that the hydroxyl group was preferentially substituted at the ortho-positions to form 6-OH-BDE47 in comparison with 5-OH-BDE47 and 3-OH-BDE47. A similar result was obtained with $\mathrm{CB} 77$ being primarily metabolized to 6-OHCB77 and 5-OH-CB77 (in a ratio of 10:1) in poplar tissues (Zhai et al., 2010a). The study of OH-PBDE metabolites in human serum samples from an electronic waste dismantling site showed that all three OH-PBDE metabolites identified were of substitution of hydroxyl groups at the ortho-positions (Yu et al., 2010).

The total concentration of OH-PBDEs in the BDE-28, -47 exposed maize roots and stems increased with increasing exposure time until 48 and $24 \mathrm{~h}$, respectively, and then decreased, whereas the total concentration of OH-PBDEs in roots and stems increased with increasing exposure time for the BDE-15 exposure, which were similar to the trend of their parent PBDE congeners in our previous study (Wang et al., 2011c). No significant difference in the total concentration of OH-PBDEs in leaves over time was
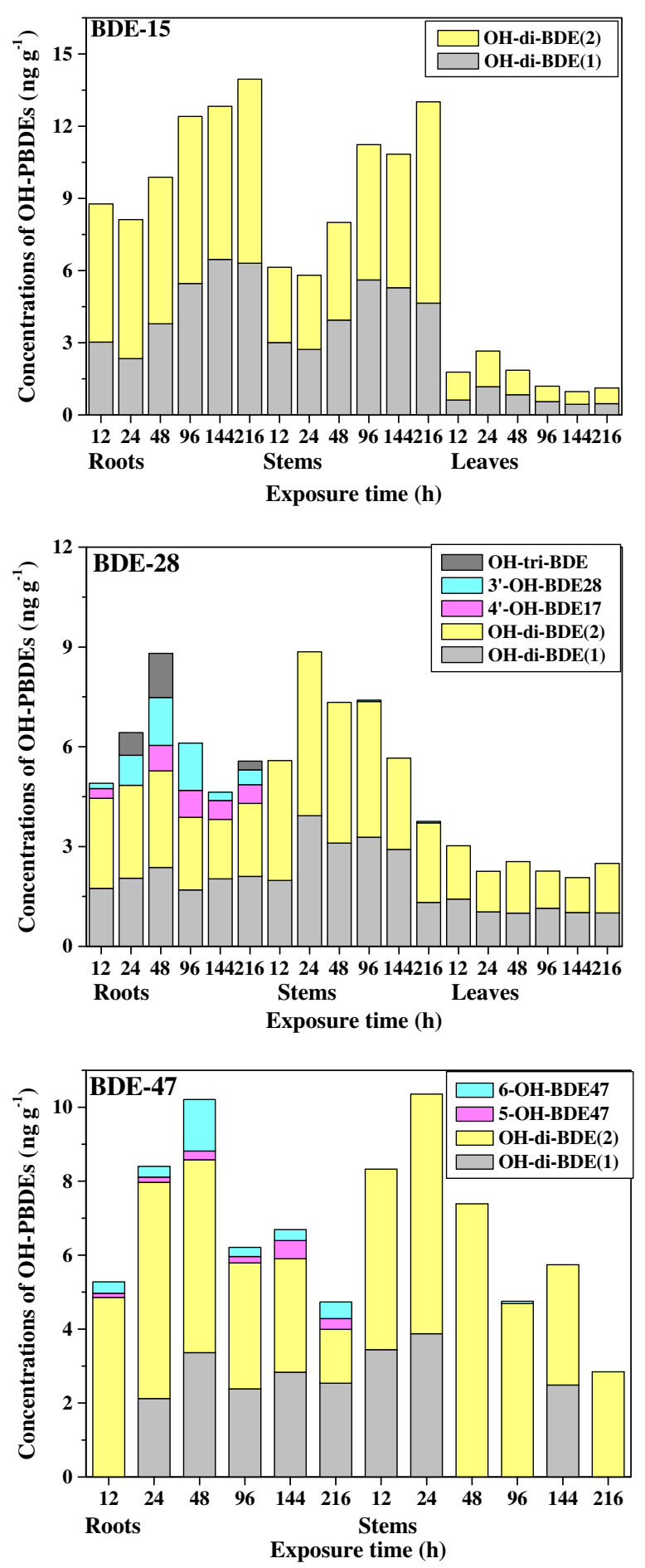

Fig. 2. Time-dependent concentrations of the hydroxylated PBDEs (OH-PBDEs) in maize.

observed between the BDE-15 and -28 exposure. Some studies have shown that the elimination half-life of 6-OH-BDE47 in rat plasma is 71.7-85.6 min (Lai et al., 2011) and much shorter than the half-life of the parent compound BDE-47 (664, 30-40, and 31 d in humans, mice and rats, respectively) (Staskal et al., 2005), and this is likely due to the insignificant accumulation 6-OHBDE47 in rats. In the present study the total concentration of OH-PBDEs in each part of maize was unlikely to increase significantly with increasing exposure time, and decreased in the BDE28 and -47 exposed maize roots and stems at the later exposure time. This result may be attributed to a combination of the short 
elimination half-life of OH-PBDEs and further metabolism of the OH-PBDEs inside maize.

Similar to the de-PBDEs, the total concentration of the $\mathrm{OH}-$ PBDEs was relatively low in leaves compared with roots and stems. Although the concentrations of the parent PBDEs (BDE-15, -28 and -47) were much higher in roots than in stems (Wang et al., 2011c), the total concentration of the OH-PBDEs between roots and stems showed no discernible difference. It might be speculated that different activities of metabolic enzymes in different plant parts contribute to the different metabolic ability (Chhikara et al., 2010; Zhai et al., 2010b).

\subsection{Methoxylated metabolites of PBDEs in maize}

Methoxylated metabolites were analyzed in maize root, stem and leaf samples after exposure to BDE-15, -28 and -47 . Four MeO-PBDEs (2'-MeO-BDE3, 3'-MeO-BDE28, 5-MeO-BDE47 and 6$\mathrm{MeO}-\mathrm{BDE} 47)$ of the seven MeO-PBDE standards were detected, and no $3^{\prime}$-MeO-BDE7, 4'-MeO-BDE17 or 3-MeO-BDE47 was detected in the plant samples (Fig. 3). Consistent with the debromination and hydroxylation results, MeO-PBDEs were also detected in maize only after exposure for $12 \mathrm{~h}$. One MeO-PBDE (2'-MeO-BDE3), two MeO-PBDEs (2'-MeO-BDE3 and $3^{\prime}-\mathrm{MeO}-\mathrm{BDE} 28$ ), and four MeO-PBDEs (2'-MeO-BDE3, 3'-MeO-BDE28, 5-MeO-BDE47 and 6MeO-BDE47) were detected in maize exposed to BDE-15, -28 and -47 , respectively. No MeO-PBDE was measured in the blank maize controls (without PBDE exposure) or exposure solutions, suggesting that MeO-PBDEs resulted from metabolism inside the plants. However, it is difficult to determine whether the MeO-PBDEs are formed by methylation of the OH-PBDEs or directly by the methoxylation of PBDEs. The concentration of 6-MeO-BDE47 was much higher than those of 3'-MeO-BDE28 and 5-MeO-BDE47, but no 3$\mathrm{MeO}-\mathrm{BDE} 47$ was detected in maize after BDE-47 exposure. This result indicates that the methoxy group might also be preferentially substituted at the ortho-positions to form 6-MeO-BDE47. Similar observations of the most frequently detected MeO-PBDE of 6MeO-BDE47 in many environmental matrices and biotic samples have also been reported previously (Malmvärn et al., 2008; Lacorte and Ilonomou, 2009).

Fig. 3 shows the time-dependent accumulation of MeO-PBDEs in maize exposed to BDE-15, -28 and -47. The total MeO-PBDE concentration was also in the order: roots $>$ stems $>$ leaves. With increasing exposure time, the total MeO-PBDE concentration in maize exposed to BDE-28 and -47 increased until $96 \mathrm{~h}$ and then decreased, whereas the total concentration of MeO-PBDEs in maize exposed to BDE-15 continued to increase. It is suggested that MeO-PBDEs would be further degraded to other metabolites in maize.

\subsection{Transformation of PBDEs, OH-PBDEs and MeO-PBDEs in maize}

Debromination, hydroxylation, and methoxylation of PBDEs in maize occurred rapidly, and all of the metabolites were detected in each plant part only after $12 \mathrm{~h}$ exposure to PBDEs. A significantly positive linear relationship was found between each parent PBDE and its hydroxylated and methoxylated metabolites over the exposure time (BDE-28 and 3'-OH-BDE28: $R=0.84, P<0.0001$; BDE-28 and $3^{\prime}-\mathrm{MeO}-\mathrm{BDE} 28$ : $R=0.51, P<0.05 ; 3^{\prime}-\mathrm{OH}-\mathrm{BDE} 28$ and $3^{\prime}-\mathrm{MeO}-$ BDE28: $R=0.56, \quad P<0.05 ; \mathrm{BDE}-47$ and $\mathrm{OH}-\mathrm{BDE} 47: \quad R=0.85$, $P<0.001$; BDE-47 and MeO-BDE47: $R=0.93, P<0.0001$; $\mathrm{OH}-$ BDE47 and MeO-BDE47: $R=0.72, P<0.001$ ), suggesting the existence of the transformation of PBDEs, OH-PBDEs and MeO-PBDEs inside maize and a similar metabolic pathway between OH-PBDEs and MeO-PBDEs. Furthermore, it was found that the bromine atoms at the ortho-positions of BDE-28 and -47 departed more
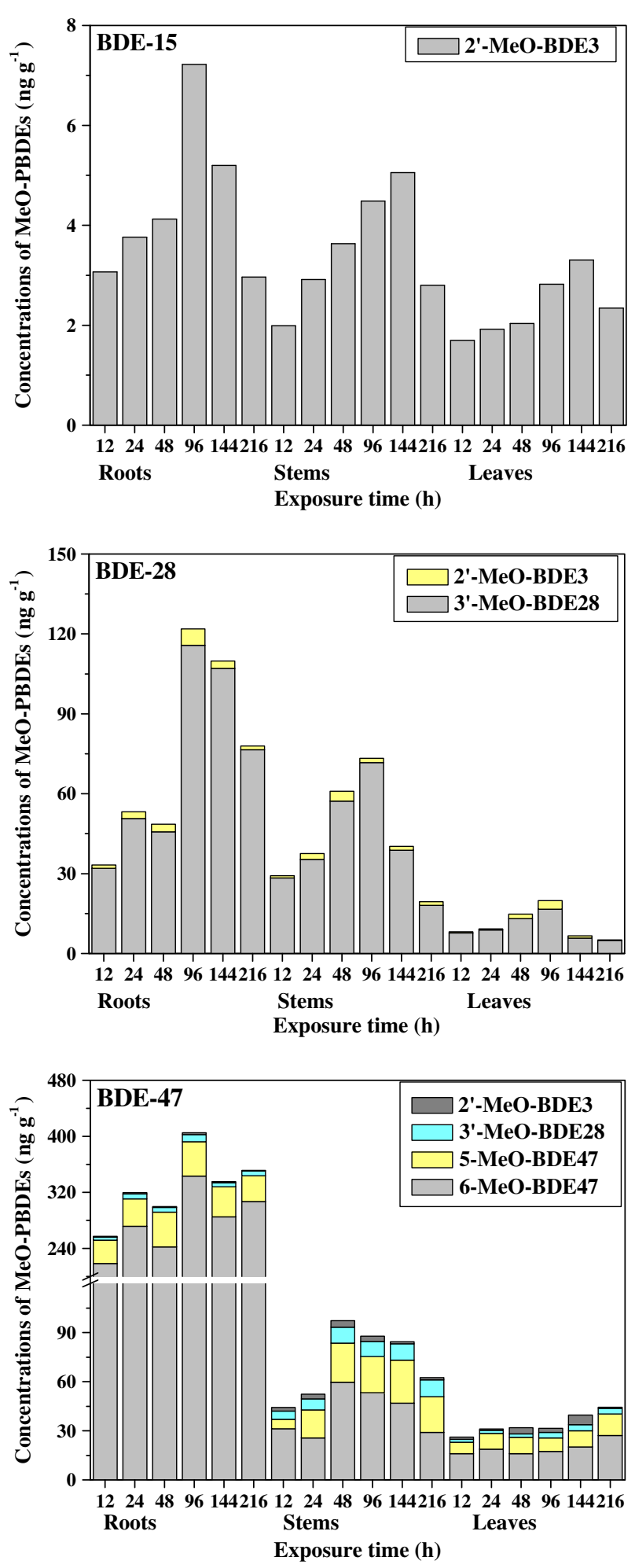

Fig. 3. Time-dependent concentrations of the methoxylated PBDEs (MeO-PBDEs) in maize.

easily than those at the para-positions, and the hydroxyl/methoxy group was also preferentially substituted at the ortho-positions.

To compare the metabolites in different plant parts expected for higher concentration in leaves than in stems after BDE-28 exposure, the total concentration of each type of the metabolites (dePBDEs, OH-PBDEs and MeO-PBDEs) was lower in leaves than in roots and stems. The concentration ratio of the total debrominated, hydroxylated or methoxylated metabolites to their parent 
Table 1

The mean ratios of the total metabolites to their parent congeners in maize roots, stems and leaves.

\begin{tabular}{llll}
\hline & Roots & Stems & Leaves \\
\hline$\sum$ de-PBDEs/BDE-15 & $0.015(0.0050)$ & $10(4.4)$ & $17(5.3)$ \\
$\sum$ de-PBDEs/BDE-28 & $0.021(0.0042)$ & $2.0(0.91)$ & $15(6.3)$ \\
$\sum$ de-PBDEs/BDE-47 & $0.0081(0.0016)$ & $27(6.1)$ & $12(5.2)$ \\
$\sum$ MeO-PBDEs/BDE-15 & $0.0010(0.00037)$ & $0.31(0.16)$ & $1.1(0.58)$ \\
$\sum$ MeO-PBDEs/BDE-28 & $0.019(0.011)$ & $3.1(2.0)$ & $5.9(4.5)$ \\
$\sum$ MeO-PBDEs/BDE-47 & $0.053(0.014)$ & $28(5.7)$ & $34(18)$ \\
$\sum$ OH-PBDEs/BDE-15 & $0.0010(0.00030)$ & $0.55(0.25)$ & $0.80(0.35)$ \\
$\sum$ OH-PBDEs/BDE-28 & $0.0014(0.00050)$ & $0.49(0.23)$ & $1.8(1.0)$ \\
$\sum$ OH-PBDEs/BDE-47 & $0.0011(0.00013)$ & $2.8(1.4)$ & -
\end{tabular}

a Data within parenthesis are standard errors.

congener in each part of maize was further calculated (Table 1 ). The ratios were in the increasing order of roots $\ll$ stems $<$ leaves for all the three types of metabolites with the only exception of the lower ratio of the debrominated metabolites in leaves than in stems after BDE-47 exposure. It is difficult to make a detailed interpretation of the metabolites in plants since accumulation of the metabolites in maize may result from a combination of metabolism and translocation of the metabolites inside the plants. Nevertheless, these findings suggest that PBDEs in maize leaves and stems are more susceptible to metabolism compared with those in the roots. The concentration ratios of the total debrominated, hydroxylated or methoxylated metabolites to the parent congener (BDE-28 or -47) in each part of maize were in the decreasing order MeO-PBDEs $>$ de-PBDEs $\gg$ OH-PBDEs with the exception of the metabolites in leaves of the BDE-28 exposed maize, for which an order of OH-PBDEs < MeO-PBDEs < de-PBDEs was obtained. However, after BDE-15 exposure, the order of de-PBDEs $\gg$ MeO-PBDE$\mathrm{s}>\mathrm{OH}-\mathrm{PBDEs}$ was obtained, possibly due to the fact that only limited MeO-PBDE standards are available to date, particularly without MeO-BDE15. The evidence that much lower concentrations of OH-PBDEs than of de-PBDEs or MeO-PBDEs in maize might be attributed to the shorter elimination half-life (Staskal et al., 2005; Kelly et al., 2008; Lai et al., 2011) and as well as less transformation of OH-PBDEs due to their greater toxicity compared with PBDEs and MeO-PBDEs.

\section{Conclusions}

The results of this study suggest that debromination, hydroxylation and methoxylation of BDE-15, -28 and -47 in maize occurred rapidly, and the metabolites might be further metabolized. Compared to para-positions, bromine atoms at the ortho-positions on the biphenyl structure departed more easily, and the introduction of a hydroxyl/methoxy group at the ortho-positions was also easier. Furthermore, it is suggested that PBDEs are more susceptible to metabolism in leaves and stems than in roots, and the total concentration of MeO-PBDEs was significantly higher than the concentration of OH-PBDEs. These results thus provide important information about the metabolic behavior of PBDEs in plants.

\section{Acknowledgments}

This work was funded by the National Basic Research Program of China (Project 2009CB421603) and National Natural Science Foundation of China (Projects 21177139 and 20921063).

\section{Appendix A. Supplementary material}

Supplementary data associated with this article can be found, in the online version, at http://dx.doi.org/10.1016/j.chemosphere. 2012.05.026.

\section{References}

Betts, K., 2008. Does a key PBDE break down in the environment? Environ. Sci. Technol. 42, 6781.

California State Assembly, 2003. Assembly Bill No. 302. California State Assembly, Sacramento, CA.

Chhikara, S., Paulose, B., White, J.C., Dhankher, O.P., 2010. Understanding the physiological and molecular mechanism of persistent organic pollutant uptake and detoxification in cucurbit species (zucchini and squash). Environ. Sci. Technol. 44, 7295-7301.

Collins, C., Fryer, M., Grosso, A., 2006. Plant uptake of non-ionic organic chemicals. Environ. Sci. Technol. 40, 45-52.

de Wit, C.A., 2002. An overview of brominated flame retardants in the environment Chemosphere 46, 583-624.

EEC, 2003. Directive 2003/11/EC of the european parliament and of the council of 6 february 2003 amending for the 24th time council directive 76/769/EEC relating to restrictions on the marketing and use of certain dangerous substances and preparations (pentabromodiphenyl ether and octabromodiphenyl ether). Off. J. Euro. Union L42, 45-46.

Eriksson, J., Green, N., Marsh, G., Bergman, Å., 2004. Photochemical decomposition of 15 polybrominated diphenyl ether congeners in methanol/water. Environ. Sci. Technol. 38, 3119-3125.

Fang, L., Huang, J., Yu, G., Wang, L.N., 2008. Photochemical degradation of six polybrominated diphenyl ether congeners under ultraviolet irradiation in hexane. Chemosphere 71, 258-267.

Gerecke, A.C., Hartmann, P.C., Heeb, N.V., Kohler, H.P.E., Giger, W., Schimid, P., Zennegg, M., Kohler, M., 2005. Anaerobic degradation of decabromodiphenyl ether. Environ. Sci. Technol. 39, 1078-1083.

Haglund, P.S., Zook, D.R., Buser, H.R., Hu, J., 1997. Identification and quantification of polybrominated diphenyl ethers and methoxy-polybrominated diphenyl ethers in Baltic biota. Environ. Sci. Technol. 31, 3281-3287.

Hassanin, A., Breivik, K., Meijer, S.N., Steinnes, E., Thomas, G.O., Jones, K.C., 2004 PBDEs in European background soils: levels and factors controlling their distribution. Environ. Sci. Technol. 38, 738-745.

He, J.Z., Robrock, K.R., Alvarez-Cohen, L., 2006. Microbial reductive debromination of polybrominated diphenyl ethers (PBDEs). Environ. Sci. Technol. 40 4429-4434.

Hites, R.A., 2004. Polybrominated diphenyl ethers in the environment and in people: a meta-analysis of concentrations. Environ. Sci. Technol. 38, 945-956.

Huang, H.L., Zhang, S.Z., Christie, P., Wang, S., Xie, M., 2010. Behavior of decabromodiphenyl ether (BDE-209) in the soil plant system: uptake, translocation, and metabolism in plants and dissipation in soil. Environ. Sci. Technol. 44, 663-670

Kelly, B.C., Ikonomou, M.G., Blair, J.D., Gobas, F.A.P.C., 2008. Hydroxylated and methoxylated polybrominated diphenyl ethers in a Canadian Arctic marine food web. Environ. Sci. Technol. 42, 7069-7077.

Kierkegaard, A., Burreau, S., Marsh, G., Klasson-Wehler, E., de Wit, C., Asplund, L., 2001. Metabolism and distribution of $2,2^{\prime}, 4,4^{\prime}$-tetrabromo $\left[{ }^{14} \mathrm{C}\right]$ diphenyl ether in pike (Esox lucius) after dietary exposure. Organohalog. Compd. 52, 58-61.

La Guardia, M.J., Hale, R.C., Harvey, E., 2007. Evidence of debromination of decabromodiphenyl ether (BDE-209) in biota from a wastewater receiving stream. Environ. Sci. Technol. 41, 6663-6670.

Lacorte, S., Ilonomou, M.G., 2009. Occurrence and congener specific profiles of polybrominated diphenyl ethers and their hydroxylated and methoxylated derivatives in breast milk from Catalonia. Chemosphere 74, 412-420.

Lai, Y.Q., Chen, X.G., Lam Michael, H.W., Cai, Z.W., 2011. Analysis of hydroxylated polybrominated diphenyl ethers in rat plasma by using ultra performance liquid chromatography-tandem mass spectrometry. J. Chromatogr. B 879, 1086-1090.

Liu, J.Y., Hu, D.F., Jiang, G.B., Schnoor, J.L., 2009. In vivo biotransformation of $3,3^{\prime}, 4,4^{\prime}$-tetrachlorobiphenyl by whole plants-poplars and switchgrass. Environ. Sci. Technol. 43, 7503-7509.

López, P., Brandsma, S.A., Leonards, P.E.G., de Boer, J., 2009. Methods for the determination of phenolic brominated flame retardants, and by-products formulation intermediates and decomposition products of brominated flame retardants in water. J. Chromatogr. A 1216, 334-345.

Malmberg, T., Athanasiadou, M., Marsh, G., Brandt, I., Bergman, Å., 2005. Identification of hydroxylated polybrominated diphenyl ether metabolites in blood plasma from polybrominated diphenyl ether exposed rats. Environ. Sci. Technol. 39, 5342-5348.

Malmvärn, A., Marsh, G., Kautsky, L., Athanasiadou, M., Bergman, Å., Asplund, L. 2005. Hydroxylated and methoxylated brominated diphenyl ethers in the red algae Ceramium tenuicorne and blue mussels from the Baltic Sea. Environ. Sci. Technol. 39, 2990-2997.

Malmvärn, A., Zebühr, Y., Kautsky, L., Bergman, Å., Asplund, L., 2008. Hydroxylated and methoxylated polybrominated diphenyl ethers and polybrominated dibenzo-p-dioxins in red alga and cyanobacteria living in the Baltic Sea. Chemosphere 72, 910-916.

Marsh, G., Athanasiadou, M., Bergman, Å., Asplund, L., 2004. Identification of hydroxylated and methoxylated polybrominated diphenyl ethers in Baltic Sea salmon (Salmo salar) blood. Environ. Sci. Technol. 38, 10-18.

Meerts, I.A.T.M., Letcher, R.J., Hoving, S., Marsh, G., Bergman, Å., Lemmen, J.G., van der Burg, B., Brouwer, A., 2001. In vitro estrogenicity of polybrominated diphenyl ethers, hydroxylated PBDEs, and polybrominated bisphenol A compounds. Environ. Health Perspect. 109, 399-407. 
Mueller, K.E., Mueller-Spitz, S.R., Henry, H.F., Vonderheide, A.P., Soman, R.S., Kinkle, B.K., Shann, J.R., 2006. Fate of pentabrominated diphenyl ethers in soil: abiotic sorption, plant uptake, and the impact of interspecific plant interactions. Environ. Sci. Technol. 40, 6662-6667.

Munschy, C., Héas-Moisan, K., Tixier, C., Pacepavicius, G., Alaee, M., 2010. Dietary exposure of juvenile common sole (Solea solea L.) to polybrominated diphenyl ethers (PBDEs): Part 2. Formation, bioaccumulation and elimination of hydroxylated metabolites. Environ. Pollut. 158, 3527-3533.

Qiu, X.H., Mercado-Feliciano, M., Bigsby, R.M., Hites, R.A., 2007. Measurement of polybrominated diphenyl ethers and metabolites in mouse plasma after exposure to a commercial pentabromodiphenyl ether mixture. Environ. Health Perspect. 115, 1052-1058.

Ren, G.F., Yu, Z.Q., Ma, S.T., Zheng, K.W., Wang, Y., Wu, M.H., Sheng, G.Y., Fu, J.M., 2010. Determination of polybrominated diphenyl ethers and their methoxylated and hydroxylated metabolites in human serum from electronic waste dismantling workers. Anal. Methods 3, 408-413.

Robrock, K.R., Korytar, P., Alvarez-Cohen, L., 2008. Pathways for the anaerobic microbial debromination of polybrominated diphenyl ethers. Environ. Sci. Technol. 42, 2845-2852.

Shih, Y.H., Wang, C.K., 2009. Photolytic degradation of polybromodiphenyl ethers under UV-lamp and solar irradiations. J. Hazard. Mater. 165, 34-38.

Sinkkonen, S., Rantalainen, A.L., Paasivirta, J., Lahtiper, M., 2004. Polybrominated methoxy diphenyl ethers (MeO-PBDEs) in fish and guillemot of Baltic, Atlantic and Arctic environments. Chemosphere 56, 767-775.

Staskal, D.F., Diliberto, J.J., DeVito, M.J., Bimbaum, L.S., 2005. Toxicokinetics of BDE 47 in female mice: effects of dose, route of exposure, and time. Toxicol. Sci. 83, $215-223$.

Tokarz, J.A., Ahn, M.Y., Leng, J., Filley, T.R., Nies, L., 2008. Reductive debromination of polybrominated diphenyl ethers in anaerobic sediment and a biomimetic system. Environ. Sci. Technol. 42, 1157-1164.

Teuten, E.L., Xu, L., Reddy, C.M., 2005. Two abundant bioaccumulated halogenated compounds are natural products. Science 307, 917-920.

Ueno, D., Darling, C., Alaee, M., Pacepavicius, G., Teixeira, C., Campbell, L., Letcher, R.J., Bergman, Å., Marsh, G., Muir, D., 2008. Hydroxylated polybrominated diphenyl ethers (OH-PBDEs) in the abiotic environment: surface water and precipitation from Ontario, Canada. Environ. Sci. Technol. 42, 1657-1664.
UNEP, United Nations Environment Programme Chemicals, 2002. Regionally based assessment of persistent toxic substances (RBA/PTSs). North America Regional Report. Global Environment Facility.

Verreault, J., Gabrielsen, G.W., Chu, S., Muir, D.C.G., Andersen, M., Hamaed, A. Letcher, R.J., 2005. Flame retardants and methoxylated and hydroxylated polybrominated diphenyl ethers in two Norwegian Arctic top predators: glaucous gulls and polar bears. Environ. Sci. Technol. 39, 6021-6028.

Wan, Y., Wiseman, S., Chang, H., Zhang, X.W., Jones, P.D., Hecker, M., Kannan, K., Tanabe, S., Hu, J.Y., Lam, M.H.W., Giesy, J.P., 2009. Origin of hydroxylated brominated diphenyl ethers: natural compounds or man-made flame retardants? Environ. Sci. Technol. 43, 7536-7542.

Wang S. Wu, T., Huang, H.L. Ping H., Lu, A.X, Zhang S.Z, 2011a. Analysis of hydroxylated polybrominated diphenyl ethers in plant samples using ultra performance liquid chromatography-mass spectrometry. Sci. China Chem. 54, 1782-1788.

Wang, S., Zhang, S.Z., Huang, H.L., Christie, P., 2011b. Behavior of decabromodiphenyl ether (BDE-209) in soil: effects of rhizosphere and mycorrhizal colonization of ryegrass roots. Environ. Pollut. 159, 749-753.

Wang, S., Zhang, S.Z., Huang, H.L., Zhao, M.M., Lv, J.T., 2011c. Uptake, translocation and metabolism of lesser polybrominated diphenyl ethers (PBDEs) and polychlorinated biphenyls (PCBs) in maize (Zea mays L.). Chemosphere 85, 379-385.

Wang, Y., Ding, W.W., Jin, J., 2011d. Determination of hydroxylated polylrominated diphenyl ethers in human serum by ultra performance liquid chromatographytandem mass spectrometry. Chin. J. Anal. Chem. 39, 22-26.

Yu, Z.O., Zheng, K.W., Ren, G.F., Zheng, Y.Y., Ma, S.T., Peng, P.G., Wu, M.H., Sheng, G.Y., Fu, J.M., 2010. Identification of hydroxylated octa- and nonabromodiphenyl ethers in human serum from electronic waste dismantling workers. Environ. Sci. Technol. 44, 3979-3985.

Zhai, G.S., Lehmler, H.J., Schnoor, J.L., 2010a. Identification of hydroxylated metabolites of $3,3^{\prime}, 4,4^{\prime}$-tetrachlorobiphenyl and metabolic pathway in whole poplar plants. Chemosphere $81,523-528$.

Zhai, G.S., Lehmler, H.J. Schnoor, J.L., 2010b. Hydroxylated metabolites of 4monochlorobiphenyl and its metabolic pathway in whole poplar plants. Environ. Sci. Technol. 44, 3901-3907. 